\title{
Electrical Dry Needling Versus Conventional Physiotherapy in the Treatment of Active and Latent Myofascial Trigger Points in Patients With Nonspecific Chronic Low Back Pain
}

\author{
Inmaculada C. Lara-Palomo ( $\square$ inma.lara.palomo@gmail.com )
}

Universidad de Almeria https://orcid.org/0000-0003-1899-2063

\section{Esther Gil-Martínez}

Deparment of nursing, physiotherapy and medicine. Almeria University

\section{Eduardo Antequera-Soler}

Department of nursisn, physiotherapy and medicine. Almeria University Adelaida María Castro-Sánchez

Department of nursing, physiotherapy and medicine. Almeria University

\section{Manuel Fernández-Sánchez}

Department of nursing, physiotherapy and medicine. Almeria University

\section{Héctor García-López}

Department of nursing, physiotherapy and medicine. Almeria University

\section{Research Article}

Keywords: back pain, Pain intensity, disability, fear of movement, quality of life, quality of sleep, anxiety and depression

Posted Date: October 18th, 2021

DOl: https://doi.org/10.21203/rs.3.rs-580221/v1

License: (c) (1) This work is licensed under a Creative Commons Attribution 4.0 International License. Read Full License

Version of Record: A version of this preprint was published at Trials on March 28th, 2022. See the published version at https://doi.org/10.1186/s13063-022-06179-y. 


\section{Abstract}

Background: Chronic low back pain is considered to be one of the main causes of absenteeism from work and primary and specialized consultations. The symptoms of non-specific chronic low back pain may be accompanied by the activation of myofascial trigger points in the muscles, together with local and/or referred pain. Electrical dry needling is increasingly used in the treatment of lumbar myofascial pain. Conventional physiotherapy, however, is a popular approach to chronic pathologies, and there is evidence of different modalities of physiotherapy being used in the treatment of chronic low back pain. The aim of this study has been to determine the effectiveness of electrical dry needling versus conventional physiotherapy when applied to active and latent myofascial trigger points in patients with nonspecific chronic low back pain.

Methods and Analysis. This is a controlled, randomized, two-arm, double-blind study. A total of 92 patients with chronic low back pain (time to onset $\geq 3$ months, Roland Morris Disability Questionnaire score $\geq 4$ ) will be recruited from the University of Almería. Participants will be divided into two study groups ( $n=40$, respectively) to receive treatment of low back pain with electrical dry needling and conventional physiotherapy (ischaemic compression, analytic stretching and postural education training dossier). A total of 3 sessions will be administered once a week for 3 weeks. Pain intensity, disability, fear of movement, quality of life, quality of sleep, anxiety and depression, pressure pain threshold, abdominal strength, and lumbar mobility will be recorded at 3 weeks (post-immediate) and 2 months after the end of treatment.

Ethics and dissemination. Ethics and Research Committee of the University of Almería (UALBI02020/044). The results of the study will made available to researchers, clinicians and health professionals through publications in international journals and presentations in conferences.

Trial registration number: NCT04804228. Recruiting

\section{Background}

Low back pain is a major public health problem in industrialized countries that causes individual suffering, absenteeism from work, and in some cases early retirement. Since it is a common pathology that is difficult to treat effectively, low back pain represents a high economic burden for both society and the health system, ${ }^{1-3}$ and is considered one of the main causes primary and specialized care consultations. ${ }^{4}$ In Europe, the direct and indirect costs of low back pain account for between $1.7 \%$ and $2.1 \%$ of the annual gross domestic product. ${ }^{8,9}$

Back pain, which affects up to $23 \%$ of the population worldwide, is the most common chronic disease in people under 65 years of age. With a lifetime prevalence of up to $85 \%$ in industrialized countries, ${ }^{3}$ estimates suggests that between $24 \%$ and $80 \%$ of patients have at least one recurrence per 
year, being more frequent and persistent in older adults. ${ }^{2,5,6} \mathrm{In}$ any given six-month period, $72 \%$ of adults in the general population will report low back pain and $11 \%$ will report disabling low back pain. ${ }^{7}$

Pain intensity, degree of pain interference with activities of daily living (resulting in disability), and healthrelated quality of life are among the primary outcomes in studies in patients with low back pain. ${ }^{1}$ In the 2010 Global Burden of Disease Study, which includes 291 diseases, low back pain ranked first in terms of disability and sixth in terms of overall burden. ${ }^{10}$ In addition to age, psychological factors such as emotional distress and dysfunctional pain coping mechanisms play an important role in the development and/or persistence of nonspecific chronic low back pain (CLBP). ${ }^{6}$

The symptoms of CLBP may be accompanied by the activation of myofascial trigger points (MTrP) in the lumbar and proximal muscles, together with local and/or referred pain. ${ }^{11,12}$ Clinically, MTrP present as palpably taut bands with a local twitch response and pain on pressure. ${ }^{12-14}$ When the points are active, digital palpation causes pain to radiate to a distant site (referred pain); when they are latent, palpation may be locally painful, but no radiation occurs (local pain). ${ }^{11,12}$

The MTrPs of each muscle have their own characteristic pain pattern; therefore, the spread of the pain can help identify the muscles that may contain active and latent trigger points. ${ }^{15}$ CLBP is associated with the presence of MTrP in the quadratus lumborum muscle, and often also in the lumbar and superficial paraspinal multifidus muscles. ${ }^{13}$

Non-invasive treatment options for CLBP remain controversial, and there is no general consensus on the best approach. ${ }^{16}$ Some trials in CLBP and electrical dry needling conclude that there is still no strong evidence to support the clinical effectiveness of electrical dry needling on LBP versus any other treatment modality. ${ }^{17-20}$

Dry needling is typically used to treat soft tissues, such as muscles, ligaments, tendons, fascia, scar tissue, peripheral nerves, and neurovascular bundles involved in a variety of neuromusculoskeletal pain syndromes. ${ }^{21,22}$ Dry needling involves the insertion of fine monofilament needles without the use of injectables, and its therapeutic effect is based on stimulating specific reactions in the target tissue. ${ }^{23-27}$ It is a relatively new treatment modality used by physical therapists around the world as part of the complex treatment of chronic musculoskeletal pain. ${ }^{23}$ The effectiveness of this approach has been confirmed in numerous studies and systematic reviews on the management of chronic lumbar MTrPs and myofascial pain. ${ }^{28-30}$ In electrical dry needling, needle electrodes are used to deliver an electric current to the taut muscle band or the pain-generating MTrP $25,26,31$ Low frequency currents are thought to improve the physiological effects of the therapy by using electrical stimulation to enhance certain physiological reactions and achieve a speedier analgesic and anaesthetic effect than that obtained with standard dry needling in patients with low back pain. ${ }^{32.33}$ Despite the popularity of electrical dry needling in clinical physiotherapy, there is insufficient scientific evidence to show its therapeutic effects in the treatment of CLBP.. ${ }^{34-36}$ 
Various treatment approaches beyond the scope of physiotherapy have been proposed to reduce the recurrence of low back pain and its associated care costs. Clinical practice guidelines provide strong evidence that cognitive behavioural therapy, exercise, spinal manipulation, and rehabilitation with various physiotherapy procedures are all moderately effective in chronic or subacute low back pain $>4$ weeks duration). ${ }^{35,36,37}$ Recent systematic reviews and meta-analyses recommend exercise therapy to improve back strength, flexibility, range of motion, and fitness in chronic low back pain. ${ }^{38-40}$ However, there is no evidence to show whether invasive approach like the electrical dry needling is more effective than a conventional physiotherapy in patients with nonspecific CLBP.

\section{Study objectives}

The objective of this randomised controlled trial is to evaluate the effectiveness of electrical dry needling versus conventional physiotherapy in the treatment of patients with nonspecific chronic lower back pain

The specific objectives are: (i) to compare the effectiveness of electrical dry needling versus conventional physiotherapy in improving pain, functionality, lumbar spine mobility and quality of life in patients with non-specific chronic low back pain, (ii) to evaluate the effect of this therapy on active myofascial trigger points in terms of the pressure tolerance threshold following electrical dry needling versus conventional physiotherapy.

\section{Methodology}

\section{Study design and ethical approval}

This is a controlled, randomized, two-arm, double-blind study comparing: (i) patients with chronic low back pain treated with electrical dry needling, (ii) patients with chronic low back pain treated with conventional physiotherapy consisting of ischaemic compression, analytical stretching and a dossier of home lumbar spine exercises. Study participants will be randomly assigned to two groups (electrical dry needling group or conventional physiotherapy group) with a 1:1 ratio.

This protocol has been drawn up following the Standard Protocol Items: Recommendations for Interventional Trials (SPIRIT) (Appendix 3). The study will be carried out in partnership with the physiotherapy department of the University of Almería. Ethical approval for this trial was granted by University of Almería Research Ethics Committee (UALBIO2020/044). The study protocol was registered in an international clinical trial registry, Clinicaltrials.gov (protocol number NCT04804228).

\section{Participants}

A total of 80 patients aged between 30 and 65 years, diagnosed with non-specific chronic low back pain lasting more than 3 months who are not currently undergoing any type of treatment will be recruited. Patients will be randomised to two treatment groups (electrical dry needling or conventional 
physiotherapy). Participants will receive treatment once a week for three weeks in the physiotherapy laboratories of the University of Almería, with a follow-up evaluation at three weeks and two months after the start of treatment. During their first visit, participants will be screened for study eligibility according to the study inclusion and exclusion criteria, and will be assessed by a therapist blinded to the interventions. After this face-to-face evaluation, patients will be randomly assigned to one of the two groups and will receive the corresponding treatment for low back pain administered two researchers trained in the techniques used. All participants will sign the informed consent form, which complies with the Declaration of Helsinki of the World Health Organization (schedule of enrolment, interventions and assessments is shown in Figure 1).

\section{Inclusion criteria}

Both male and female patients aged between 30 and 65 years with chronic low back pain lasting 3 months or more, with a low back pain disability score $\geq 4$ on the Roland-Morris disability questionnaire (RMQ), and not receiving any other physiotherapy treatment are eligible for inclusion.

\section{Exclusion criteria}

Patients with sensory and/or coagulation disorders, a history of spinal surgery, heart complications, concurrent severe central or peripheral nervous system disease, epilepsy, needle phobia, serious pathologies that can be the main cause of chronic low back pain (for example, presence of lumbar stenosis, spondylolisthesis, tumours, etc.), or patients contraindicated for transcutaneous electrical stimulation (TENS) will be excluded.

\section{Randomization and blinding}

Subjects will be randomised to two groups using a computer-generated (Epidat 4.2) table of random numbers generated. After randomization, participants will be assigned to either the experimental electrical dry needling group or the conventional physiotherapy control group in a ratio of 1:1. Randomization will be performed by the principal investigator.

There will be 40 participants in each group. The randomly generated group allocations will be placed in sealed opaque envelopes before being delivered to the participants and stored in locked cabinets.

The outcome assessor and study statistician will be blinded to the entire process. The outcome assessor will make no attempt to guess the participant's treatment group. The computer-generated outcome measures transmitted to the statistician will not contain any information that identifies the patient's group.

\section{Interventions}

After the initial evaluation, 80 patients with CLBP will be randomly assigned to one of the two groups and will receive electrical dry needling (experimental group) or conventional physiotherapy (control group). All 
participants will receive 1 session per week for 3 weeks, until they have received a total of 3 sessions. Patients must complete $100 \%$ of their scheduled face-to-face treatment sessions, and those in the control group must also complete $80 \%$ of the home exercise sessions in order to remain in the intention-to-treat analysis.

During the study, participants can only receive their assigned treatment; they cannot combine the study treatment with medications or any other treatment. Any interference in the treatment will be grounds for exclusion. Patients may abandon the study at any time, and the assigned interventions may be suspended or modified in a particular trial patient in response to improvement or deterioration (adverse effects) of low back pain. Adverse events will be reported to the principal investigator, who will monitor the affected patients and the possible causes of these events.

\section{Electrical dry needling group}

Patients assigned to the electrical dry needling group $(n=40)$ will receive up to three 30-minute treatment sessions ( 1 session per week for 3 weeks). Electrical stimulation will be applied bilaterally to the active and latent myofascial trigger points of the following muscles, following the MTrPs maps described by Travell and Simons: ${ }^{2,14}$ quadratus lumborum, multifidus and iliocostalis. The number of needle insertion sites will vary in each patient; the treating therapist will determine the points to be treated in each session based on whether they are active, latent or absent. Prior to needle insertion, the site will be sterilized with $70 \%$ alcohol using a cotton swab (Figures 3 ).

Two sizes of sterilized disposable stainless steel acupuncture needles will be used: $0.25 \mathrm{~mm} \times 30 \mathrm{~mm}$ or $0.30 \mathrm{~mm} \times 40 \mathrm{~mm}$. The size of the needle will depend on the patient's physical constitution (i.e. muscle and/or connective tissue thickness). The needle will be inserted until it reaches the active or latent MTrP or taut band that causes the local twitch response. ${ }^{41}$ The needles will then be connected to an electric current and left in situ for 30 minutes (TensMed S82-Enraf Nonius). ${ }^{42,43}$ A low frequency current $(2 \mathrm{~Hz})$ will be generated by a TENS device with a moderate pulse duration ( 250 microseconds) and a continuous biphasic waveform at an intensity described by the patient as "mild to moderate". ${ }^{44}$

\section{Conventional physiotherapy group}

Patients assigned to the conventional physiotherapy group $(n=40)$ will receive ischaemic compression and analytical stretching of the quadratus lumborum, multifidus and iliocostal muscles once a week for a total of 3 weeks.

Ischaemic compression will consist of constant pressure stimulation with the thumb on each MTrP for between $30 \mathrm{~s}$ and $2 \mathrm{~min}$. This compression sequence will be repeated several times. The intensity of the pressure will be adjusted to a level at which each subject reports "comfortable pain", in order words, between the pain threshold and the maximum tolerable pain. ${ }^{46,47}$ See appendix 1 for the analytical stretching procedure. 
These patients will also be given a dossier of home lumbar spine exercises to be performed 5 days a week for a total of 3 weeks (Appendix 2. Dossier of home exercises). To monitor compliance, patients will be instructed to note down in a booklet the dates on which they complete the exercises in the dossier.

\section{Data collection}

At the beginning of the study, the following demographic data will be collected: age, sex, weight, height, education, and clinical presentation. Primary and secondary outcome measures will be evaluated at baseline prior to randomisation to different groups. This will be followed by an immediate post-treatment assessment (one day after the final intervention), and an evaluation two months after the end of the intervention (short-term follow-up).

\section{Primary Outcome Measures:}

- Roland Morris Disability Questionnaire (RMDQ). This self-reported questionnaire consists of 24 items that rate limitations in different activities of daily life attributed to low back pain, such as walking, bending over, sitting, lying down, dressing, sleeping, personal care and daily activities. Disability is rated from 0 points (best) to 24 points (worst). ${ }^{48}$

- Oswestry Disability Index (ODI). The Oswestry Disability Index assesses limitations in activities of daily living in 10 dimensions, each rated on a 6-point scale (0-5 points). The higher the score, the greater the disability. The overall score is expressed as a percentage and is used to classify people as minimally disabled (0\%-10\%), moderately disabled (20\%-40\%), severely disabled $(40 \%-60 \%)$, crippling back pain (60\%-80\%), or bedridden (80\%-100\%). ${ }^{49}$

- Visual Analogue Scale (VAS). Study subjects will indicate the intensity of their pain on a $100 \mathrm{~mm}$ VAS. They are asked to situate their pain on a $100 \mathrm{~mm}$ horizontal line, where $0 \mathrm{~mm}$ indicates "no pain" and $100 \mathrm{~mm}$ indicates "the worst pain imaginable". ${ }^{50}$

\section{Secondary outcome measures:}

- Quality of life using the SF-36 questionnaire. The SF-36 is a short-form, multipurpose health survey with only 36 questions. The instrument contains eight subscales (physical function, physical role, body pain, general health, vitality, social function, emotional role, and mental health) and two summary scores: physical and mental health. Scores range from $0 \%$ to $100 \%$ and indicate selfperceived health-related quality of life. ${ }^{51,52}$

- Tampa Scale for kinesiophobia (TSK). This is a 17-item questionnaire that measures fear of movement and (re) injury. Patients rate their beliefs about their kinesiophobia on a 4-point scale ranging from strongly disagree to strongly agree. ${ }^{53,54}$

- Pittsburgh Sleep Quality Index (PSQI). This is a 10-item questionnaire with a total of 19 questions related to sleep habits in the previous month. The questions are divided into 7 areas, each with a score of between 0 and 3 points. The overall score ranges from 0 (no difficulty sleeping) to 21 points (severe difficulty sleeping). ${ }^{55}$ 
- Hospital Anxiety and Depression Scale (HADS). This scale consists of 14 items related to emotional distress (anxiety, depression) in populations suffering from a physical illness. It consists of two subscales (HADA: anxiety and HADD: depression) with seven items each that score from 0 (normal) to 3 (abnormal). ${ }^{56.57}$

- McQuade test. This test evaluates the isometric resistance of the flexor muscles of the trunk. The patient is placed supine and asked to flex the head and shoulders until the scapula is lifted off the table. The number of seconds they hold that position is recorded. ${ }^{58}$

- Anterior Trunk Flexion. Standing, with legs straight, the patient is asked to bend forward and attempt to touch the ground. They are told to stop when pain or limitation of movement appear. The distance, in $\mathrm{cm}$, between the fingers and the ground is measured. ${ }^{59}$

- Spinal Mouse ${ }^{\circledR}$. This is a safe, practical and easy-to-use instrument to measure the curvature of the spine in the frontal and sagittal planes and to assess the segmental mobility of the lumbar region. ${ }^{60}$

- Pressure Algometry (Wagner FPI 10 Algometer) in MTrPs. The algometer consists of a rubber tip and a dial that measures the pressure applied to the MTrP in increments of $0.5 \mathrm{~kg}$. The pressure pain threshold will be assessed following the illustrations published by Travell and Simons. ${ }^{14}$

\section{Sample size}

The sample size was calculated according to the specifications established by Willian. ${ }^{61}$ The calculations were based on the detection of differences of 2.5 points in the RMDQ (minimum detectable difference between means for a variance of 10 points in patients with chronic low back pain), assuming a standard deviation of 2.5 points, a 2-tailed test, an alpha (a) of 0.05 and a target power (beta) of $85 \%$. The estimated target sample size has been calculated at 40 subjects per group.

The following specifications will be considered: $a=0.05$, statistical power of $85 \%$ and loss to follow-up of $10 \%$. The sample size calculation yielded a total of 92 participants to be randomized to two intervention groups.

\section{Data analysis}

Statistical analysis will be performed using SPSS@ version 21.0 and STATA 14 using the principles of intention to treat. Comparisons will be made between the two study arms. We will calculate the difference between groups after the final treatment session and at two month post-intervention (short-term results).

The efficacy variable for this clinical trial is the difference between continuous variables (i.e., RMDQ, ODI, VAS, TSK, SF-36, PSQI, HADS, pressure algometry, McQuade test, and trunk range of movement) at baseline and at predetermined time points (electric dry needling treatment vs conventional physiotherapy):

The Kolmogorov-Smirnov test will be used to assess the normality of continuous variables. 
The equality of means of the intragroup hypotheses will be analysed using the Student's t test for paired clinical variables in the case of parametric distributions and the Kruskal-Wallis $\mathrm{H}$ test in the case of nonparametric distributions.

One-way analysis of variance (ANOVA) will be used to test the intragroup hypothesis in the case of parametric distributions, and the Kruskal-Wallis $\mathrm{H}$ test will be used in nonparametric distributions.

Post hoc analysis will be obtained for parametric distributions, and Mann-Whitney $\mathrm{U}$ for nonparametric distributions.

The confidence interval will be set at $95 \%$ and the level of significance at 0.05 .

\section{Adverse effects}

No potential risks have been described so far, given that these can be prevented by the operator's knowledge of anatomy, training and experience. ${ }^{62}$ Researchers will notify study subjects of possible adverse events in the informed consent and record any adverse events that occur over the course of the study. If such events are observed, the frequency of occurrence will be analysed between groups, and if patients have any questions or require additional information about any symptoms, they will be able to contact the principal investigator by phone or email.

\section{Ethics and dissemination.}

All participants will receive verbal and written information about the study before giving their consent to participate. They will be informed that they can leave the study at any time. Participants who agree to take part in the study will sign two copies of the informed consent form, one for the research and evaluation team and one for the participant.

All hard copies will be confidential and stored in a locked filing cabinet in the research group office and in electronic format in a password-protected database. The research team will monitor the integrity of the trial data. All participants, group assignments, treatment records sociodemographic data will be coded, and the results of the questionnaires will be scored.

The data collected on each participant will be kept under lock and key by the evaluator. If the data are in digital format, they will be stored in a computer with a secret access code known only to the evaluator.

The eligibility criteria, results, and analyses will not be modified once the first participant has been enrolled in the study. Any amendment to the protocol, including changes in the eligibility criteria, the results or the analyses, will be communicated to the Institutional Research Committee of the University of Almería and reported in articles and presentations disseminating the results of the trial.

The feasibility results will be published in peer-reviewed journals and presented at academic, clinical, and health services conferences. 


\section{Discussion And Conclusions}

Although physiotherapy with dry needling and electrical dry needling have proven positive effects on chronic low back pain,22-31 the results of studies into the duration of the analgesic effect and the dose required, for example, are contradictory.63,64 Therefore, further research is required to evaluate the specific components of the treatments administered by physical therapists. This study can contribute to our understanding of the effectiveness of electrical dry needling versus conventional physiotherapy in patients with short-term non-specific chronic low back pain. The results can help physiotherapists understand whether low back pain treated with electrical dry needling can significantly reduce disability and absenteeism due to chronic low back pain. Improving chronic low back pain without absenteeism will reduce labour costs and waiting lists for rehabilitative physiotherapy. Due to the growing prevalence of chronic conditions such as low back pain and their impact on individuals, their circumstances, and society in general, it is becoming increasingly important to provide evidence-based, cost-effective interventions.65,66 These intervention must first be designed, adapted, and tested to determine their feasibility and cost before being evaluated in a high quality effectiveness trial. The trial design will be reviewed based on the findings of this study before performing a definitive trial. Timeline Patients will be recruited between May 2021 and August 2021. The study is expected to be completed in November 2021. Data analysis, writing of the scientific manuscript and submission to peer-reviewed scientific journals will take place from January 2021. A summary of the study outline is shown in Figure 3.

\section{Strengths and limitations of this study:}

This study can contribute to our understanding of the effectiveness of electrical dry needling versus conventional physiotherapy in patients with short-term non-specific chronic low back pain.

The results can help physiotherapists understand whether low back pain treated with electrical dry needling can significantly reduce disability and absenteeism due to chronic low back pain.

\section{Abbreviations}

LBP: low back pain; MTrP: Myofascial trigger points; Hz: Hertz; ODI: Oswestry Disability Index; RMDQ: Roland-Morris Low Back and Disability Questionnaire; SF-36: short-format health survey questionnaire; PSQI: Pittsburgh Sleep Quality Scale; HADS: hospital anxiety and depression scale; SPSS: statistical software package for the social sciences; TENS: Transcutaneous electrical nerve stimulation; TSK: Tampa scale of kinesiophobia; VAS: visual analogue scale.

\section{Declarations}

Funding This trial was conducted with no external funding, and its costs have been assumed by the researchers.

Availability of data and materials. Not applicable. 
Acknowledgements. Not applicable.

Conflict of interests. The authors have no conflict of interest to declare.

Patient consent for publication. Not applicable.

Ethical approval and consent to participate . This study complies with the Helsinki guidelines for human research and has been approved by the Human Research and Local Ethics Committee of the University of Almería. Participants can only be included in the study after informed consent has been obtained.

Contributor Information. ICLP: Conceptualization, methodology, writing-original draft, writing-review and editing, supervision, and project administration. HGL: Conceptualization, methodology, investigation, formal analysis, and writing-review and editing. EGM, EAS, AMCS and MFS: Conceptualization, methodology, and writing-review and editing. All authors read and approved the final manuscript.

\section{References}

1. Scholich SL, Hallner D, Wittenberg RH, et al. The relationship between pain, disability, quality of life and cognitive-behavioural factors in chronic back pain. Disabil Rehabil. 2012;34(23):1993-2000.

2. Ekman M, Johnell O, Lidgren L. The economic cost of low back pain in Sweden in 2001. Acta Orthop. 2005;76(2):275-84.

3. Becker A, Held H, Redaelli M, et al. Low back pain in primary care: costs of care and prediction of future health care utilization. Spine (Phila Pa 1976). 2010;35(18):1714-20.

4. Seguí DM, Gérvas J. El dolor lumbar. Semergen. 2002;28(1):21-41.

5. Will JS, Bury DC, Miller JA. Mechanical Low Back Pain. Am Fam Physician. 2018;98(7):421-8.

6. Waxman SE, Tripp DA, Flamenbaum R. The mediating role of depression and negative partner responses in chronic low back pain and relationship satisfaction. J Pain. 2008;9(5):434-42.

7. Cassidy JD, Côté P, Carroll LJ, et al. Incidence and course of low back pain episodes in the general population. Spine (Phila Pa 1976). 2005;30(24):2817-23.

8. GBD 2015 Disease and Injury Incidence and Prevalence Collaborators. Global, regional, and national incidence, prevalence, and years lived with disability for 310 diseases and injuries, 1990-2015: a systematic analysis for the Global Burden of Disease Study 2015. Lancet. 2016;388:1545-602.

9. Van Tulder MW, Koes BW, Bouter L. A cost-of-illness study of back pain in The Netherlands. Pain. 1995;62:233-40.

10. Hoy D, March L, Brooks P, et al. The global burden of low back pain: estimates from the Global Burden of Disease 2010 study Annals. of the Rheumatic Diseases. 2014;73:968-74.

11. Chiarotto A, Clijsen R, Fernandez-de-Las-Penas C, et al. Prevalence of Myofascial Trigger Points in Spinal Disorders: A Systematic Review and Meta-Analysis. Arch Phys Med Rehabil. 2016;97(2):31637. 
12. Rivner MH. The neurophysiology of myofascial pain syndrome. Curr Pain Headache Rep. 2001;5(5):432-40.

13. Robert D, Gerwin RD. Classification, epidemiology, and natural history of myofascial pain syndrome. Curr Pain Headache Rep. 2001;5(5):412-20.

14. Simons DG, Travell JG, Simons LS. The Trigger Point Manual. edn 2. Vol. 1. Baltimore: Lippincott Williams and Wilkins; 1998.

15. Borg Stein J, Simons DG. Myofascial pain. Arch Phys Med Rehabil. 2002;83(1):40-7.

16. Qaseem A, Wilt TJ, McLean RM, et al. Clinical Guidelines Committee of the American College of Physicians. Noninvasive Treatments for Acute, Subacute, and Chronic Low Back Pain: A Clinical Practice Guideline From the American College of Physicians. Ann Intern Med. 2017;166(7):514-30.

17. Hosseini L, Shariat A, Ghaffari MS, et al. The effect of exercise therapy, dry needling, and nonfunctional electrical stimulation on radicular pain: a case report. J Exerc Rehabil. 2018;14(5):864-9.

18. Rainey CE. The use of trigger point dry needling and intramuscular electrical stimulation for a subject with chronic low back pain: a case report. Int J Sports Phys Ther. 2013;8(2):145-61.

19. Kong JT, Puetz C, Tian L, et al. Effect of Electroacupuncture vs Sham Treatment on Change in Pain Severity Among Adults With Chronic Low Back Pain: A Randomized Clinical Trial. JAMA Netw Open. 2020;3(10):e2022787.

20. Heo I, Hwang MS, Hwang EH, et al. Electroacupuncture as a complement to usual care for patients with non-acute low back pain after back surgery: a pilot randomised controlled trial. BMJ Open. 2018;8(5):e018464.

21. Dunning J, Butts R, Henry N, et al. Electrical dry needling as an adjunct to exercise, manual therapy and ultrasound for plantar fasciitis: A multi-center randomized clinical trial. PLoS One. 2018;13(10):e0205405.

22. Dunning J, Butts $\mathrm{R}$, Zacharko $\mathrm{N}$, et al. Spinal manipulation and perineural electrical dry needling in patients with cervicogenic headache: a multicenter randomized clinical trial. Spine J. 2021;21(2):284-95.

23. Casanueva B, Rivas $P$, Rodero $B$, et al. Short-term improvement following dry needle stimulation of tender points in fibromyalgia. Rheumatol Int. 2014;34(6):861-6.

24. Manheimer E, White A, Berman B, et al. Meta-analysis: acupuncture for low back pain. Ann Intern Med. 2005;142(8):651-63.

25. Gunn CC, Milbrandt WE, Little AS, et al. Dry Needling of Muscle Motor Points for Chronic Low-Back Pain. Spine. 1980;5(3):279-91.

26. Lewit K. The needle effect in the relief of myofascial pain. Pain. 1979;6(1):83-90.

27. Dunning J, Butts R, Mourad F, et al. Dry needling: a literature review with implications for clinical practice guidelines. Phys Ther Rev. 2014;19(4):252-65. 
28. Macdonald AJ, Macrae KD, Master BR, et al. Superficial acupuncture in the relief of chronic low back pain. Ann R Coll Surg Engl. 1983;65(19):44-6.

29. Ceccherelli F, Rigoni MT, Gagliardi G, et al. Comparison of superficial and deep acupuncture in the treatment of lumbar myofascial pain: a double-blind randomized controlled study. Clin J Pain. 2002;18(3):149-53.

30. Furlan $A D$, van Tulder $M$, Cherkin $D$, et al. Acupuncture and dry-needling for low back pain: an updated systematic review within the framework of the cochrane collaboration. Spine. 2005;30(8):944-63.

31. Kumnerddee W, Pattapong N. Efficacy of electro-acupuncture in chronic plantar fasciitis: a randomized controlled trial. Am J Chin Med. 2012;40(6):1167-76.

32. Kwon $Y$, Kang M, Son $S$, et al. Different frequencies of electroacupuncture modified the cellular activity of serotonergic neurons in brainstem. Am Journal Chinese Medicine. 2000;28:435e41.

33. Zhang R, Lao L, Ren K, et al. Mechanisms of acupuncture-electroacupuncture on persistent pain. Anesthesiology. 2014;120(2):482-503.

34. Qaseem A, Wilt TJ, McLean RM, et al. Clinical Guidelines Committee of the American College of Physicians. Noninvasive Treatments for Acute, Subacute, and Chronic Low Back Pain: A Clinical Practice Guideline From the American College of Physicians. Ann Intern Med. 2017;166(7):514-30.

35. Chou R, Huffman LH, American Pain Society. American College of Physicians. Nonpharmacologic therapies for acute and chronic low back pain: a review of the evidence for an American Pain Society/American College of Physicians clinical practice guideline. Ann Intern Med. 2007;147(7):492-504.

36. França FR, Burke TN, Caffaro RR, et al. Effects of muscular stretching and segmental stabilization on functional disability and pain in patients with chronic low back pain: a randomized, controlled trial. $J$ Manipulative Physiol Ther. 2012;35(4):279-85.

37. Takamoto K, Bito I, Urakawa S, et al. Effects of compression at myofascial trigger points in patients with acute low back pain: A randomized controlled trial. Eur J Pain. 2015;19(8):1186-96.

38. Owen PJ, Miller CT, Mundell NL, et al. Which specific modes of exercise training are most effective for treating low back pain? Network meta-analysis. Br J Sports Med. 2020;54(21):1279-87.

39. Shirado O, Doi T, Akai M, et al. Multicenter randomized controlled trial to evaluate the effect of homebased exercise on patients with chronic low back pain: the Japan low back pain exercise therapy study. Spine. 2010;35:E811-9.

40. Ferreira ML, Ferreira PH, Latimer J, et al. Comparison of general exercise, motor control exercise and spinal manipulative therapy for chronic low back pain: a randomized trial. Pain. 2007;131:31-7.

41. Kalichman L, Vulfsons S. Dry needling in the management of musculoskeletal pain. J Am Board Fam Med. 2010;23(5):640-6.

42. Couto C, de Souza IC, Torres IL, Fregni F, Caumo W. Paraspinal stimulation combined with trigger point needling and needle rotation for the treatment of myofascial pain: a randomized shamcontrolled clinical trial. Clin J Pain. 2014;30(3):214-23. 
43. Tousignant-Laflamme Y, Pt PhD, Laroche C, Pt BC, Pt, Bouchard AJ, Pt BS, Pt, Michaud-Létourneau M, Pt. A randomized trial to determine the duration of analgesia following a 15- and a 30-minute application of acupuncture-like TENS on patients with chronic low back pain. Physiother Theory Pract. 2017;33(5):361-9.

44. Sluka KA, Walsh D. Transcutaneous electrical nerve stimulation: Basic science mechanisms and clinical effectiveness. J Pain. 2003;4:109-21.

45. Takamoto K, Bito I, Urakawa S, et al. Effects of compression at myofascial trigger points in patients with acute low back pain: A randomized controlled trial. Eur J Pain. 2015;19(8):1186-96.

46. Cagnie B, Castelein B, Pollie F, et al. Evidence for the Use of Ischemic Compression and Dry Needling in the Management of Trigger Points of the Upper Trapezius in Patients with Neck Pain: A Systematic Review. Am J Phys Med Rehabil. 2015;94(7):573-83.

47. Roland M, Fairbank J. The Roland-Morris Disability Questionnaire and the Oswestry Disability Questionnaire. Spine (Phila Pa 1976). 2000;25:3115-24.

48. Chiarotto A, Maxwell LJ, Terwee CB, et al. Roland-Morris Disability Questionnaire and Oswestry Disability Index: Which Has Better Measurement Properties for Measuring Physical Functioning in Nonspecific Low Back Pain? Systematic Review and Meta-Analysis. Phys Ther. 2016;96(10):162037.

49. Crichton N. Visual analogue scale (VAS). J Clin Nurs. 2001;10(5):706-6.

50. Ware JE. SF-36 health survey update. Spine (Phila Pa 1976). 2000;25(24):3130-9.

51. Alonso J, Prieto L, Antó JM. [The Spanish version of the SF-36 Health Survey (the SF-36 health questionnaire): an instrument for measuring clinical results]. Med Clin (Barc). 1995;104(20):771-6.

52. Kori SH, Miller RP, Todd DD. Kinesiophobia: a new view of chronic pain behavior. Pain Manag. 1990;3:35-43.

53. Swinkels-Meewisse EJ, Swinkels RA, Verbeek AL, et al. Psychometric properties of the Tampa Scale for kinesiophobia and the fear-avoidance beliefs questionnaire in acute low back pain. Man Ther. 2003;8:29-36.

54. Macías Fernández JA, Royuela Rico A. La versión española del índice de calidad de sueño de Pittsburgh. Inform Psiquiatr. 1996;146:465-72.

55. Montazeri A, Vahdaninia M, Ebrahimi M, et al. The Hospital Anxiety and Depression Scale (HADS): translation and validation study of the Iranian version. Health Qual Life Outcomes. 2003;1:14.

56. Herrero MJ, Blanch J, Peri JM, et al. A validation study of the hospital anxiety and depression scale (HADS) in a Spanish population. Gen Hosp Psychiatry. 2003;25(4):277-83.

57. McGuill SM, Childs A, Liebenson C. Endurace times for low back stabilization exercises: clinical targets for testing and training from a normal database. Arch Phys Med Rehabil. 1999;80:941-4.

58. Frost M, Sutckey S, Samelley LA, et al. Reliability of measuring trunk motions in centimetres. Phys Ther. 1982;62:1431-8. 
59. Livanelioglu A, Kaya F, Nabiyev V, et al. The validity and reliability of "Spinal Mouse" assessment of spinal curvatures in the frontal plane in pediatric adolescent idiopathic thoraco-lumbar curves. Eur Spine J. 2016;25(2):476-82.

60. Willian AR. Analysis, sample size, and power for estimating incremental net health benefit from clinical trial data. Control Clin Trials. 2001;22(3):228-37.

61. Halle JS, Halle RJ. Pertinent dry needling considerations for minimizing adverse effects - Part two. Int J Sports Phys Ther. 2016;11(5):810-9.

62. Wu LC, Weng PW, Chen $\mathrm{CH}$, et al. Literature Review and Meta-Analysis of Transcutaneous Electrical Nerve Stimulation in Treating Chronic Back Pain. Reg Anesth Pain Med. 2018;43(4):425-33.

63. Khadilkar A, Milne S, Brosseau L, et al. Transcutaneous electrical nerve stimulation for the treatment of chronic low back pain: a systematic review. Spine (Phila Pa 1976). 2005;30(23):2657-66.

64. Hurley DA, Hall AM, Currie Murphy L, et al. SOLAS Trial Team. Theory-driven group-based complex intervention to support self-management of osteoarthritis and low back pain in primary care physiotherapy: protocol for a cluster randomised controlled feasibility trial (SOLAS). BMJ Open. 2016;6(1):e010728.

65. Airaksinen O, Brox JI, Cedraschi C, et al, COST B13 Working Group on Guidelines for Chronic Low Back Pain. Chapter 4. European guidelines for the management of chronic nonspecific low back pain. Eur Spine J. 2006;15(2):192-300.

\section{Figures}


Patient Enrollment: Patients who are interested in participating will receive information about the study and, if they wish, will be informed of the date of the appointment with the time and place for an evaluation with the investigator.
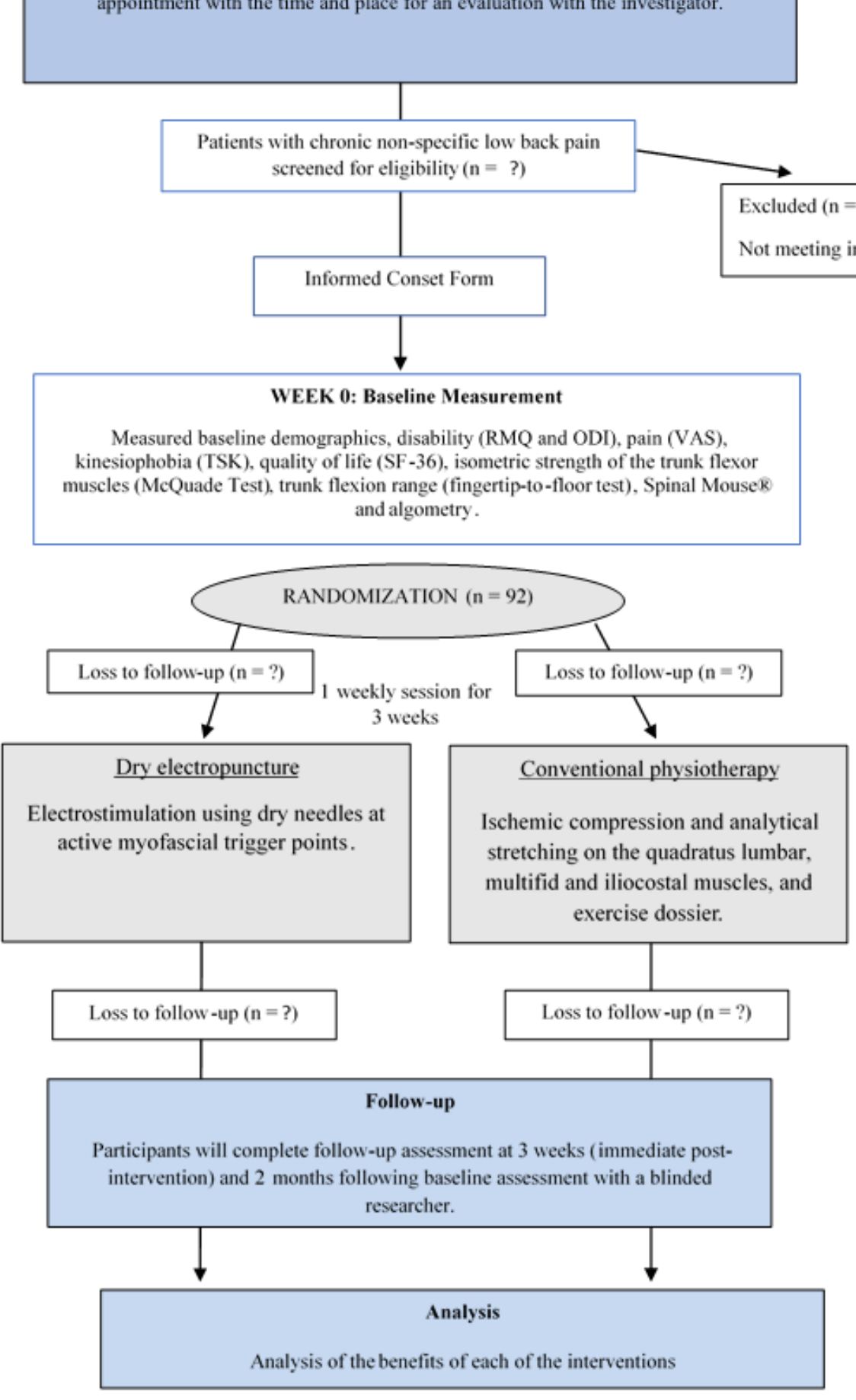

\section{Figure 1}

Design and flow of participants through the trial. *Abbreviations: RMQ = Roland-Morris Low Back and Disability Questionnaire; ODI = Oswestry Disability Index; VAS = Visual Analogue Scale; TSK = Tampa Scale for Kinesiophobia 


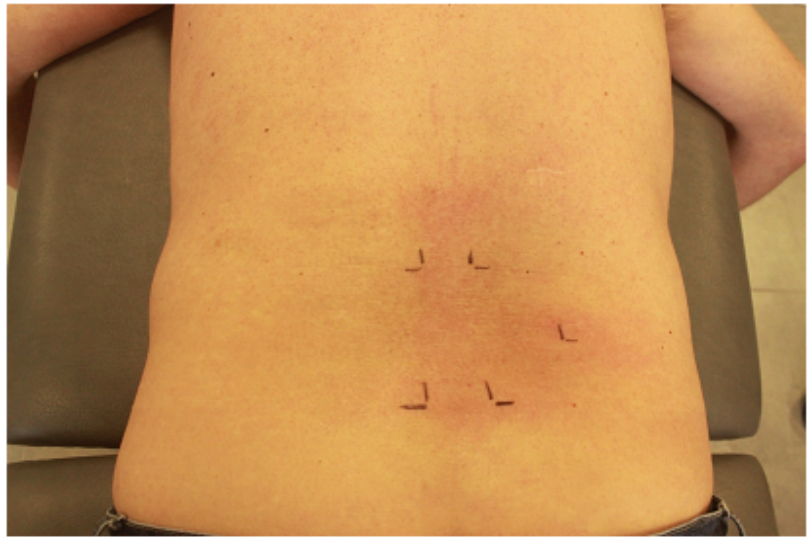

* Location and signaling of active and latent MTrP.

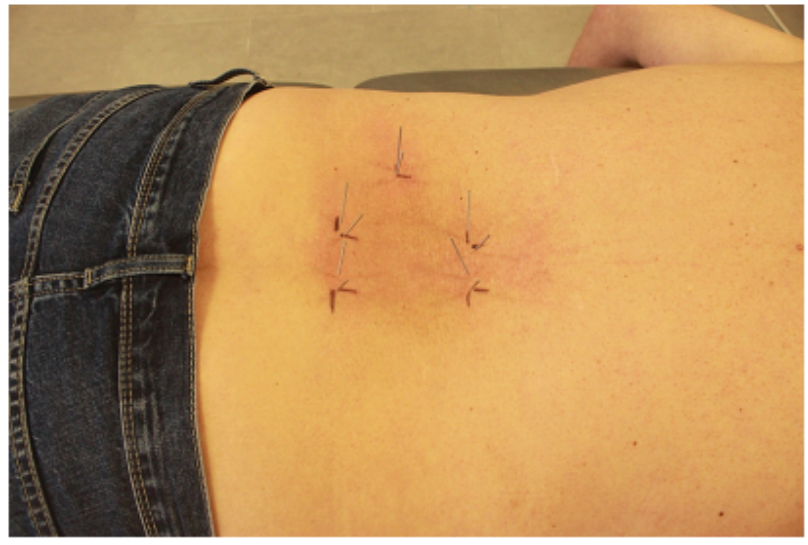

* Placement of dry needles in active and latent MTrP.

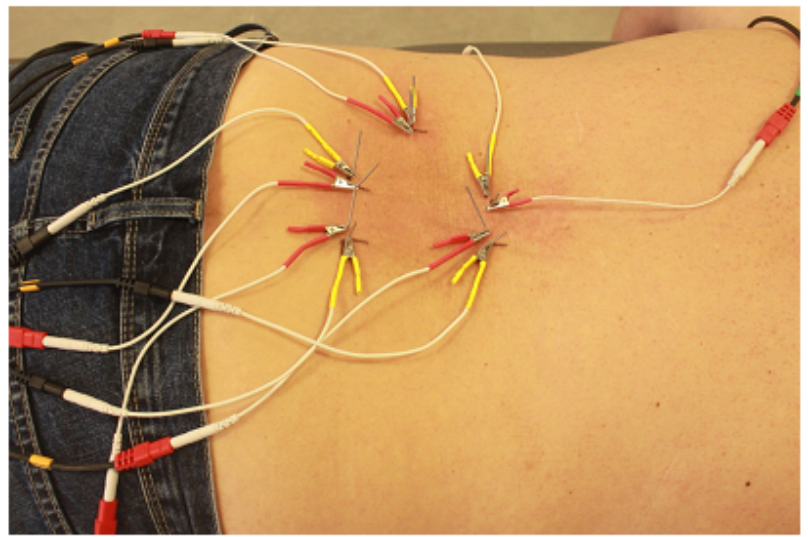

* Dry electrical needling technique in active and latent MTrPs.

Abbreviation: Myofascial Trigger Point (MTrP)

\section{Figure 2}

Electrical dry needling group. 


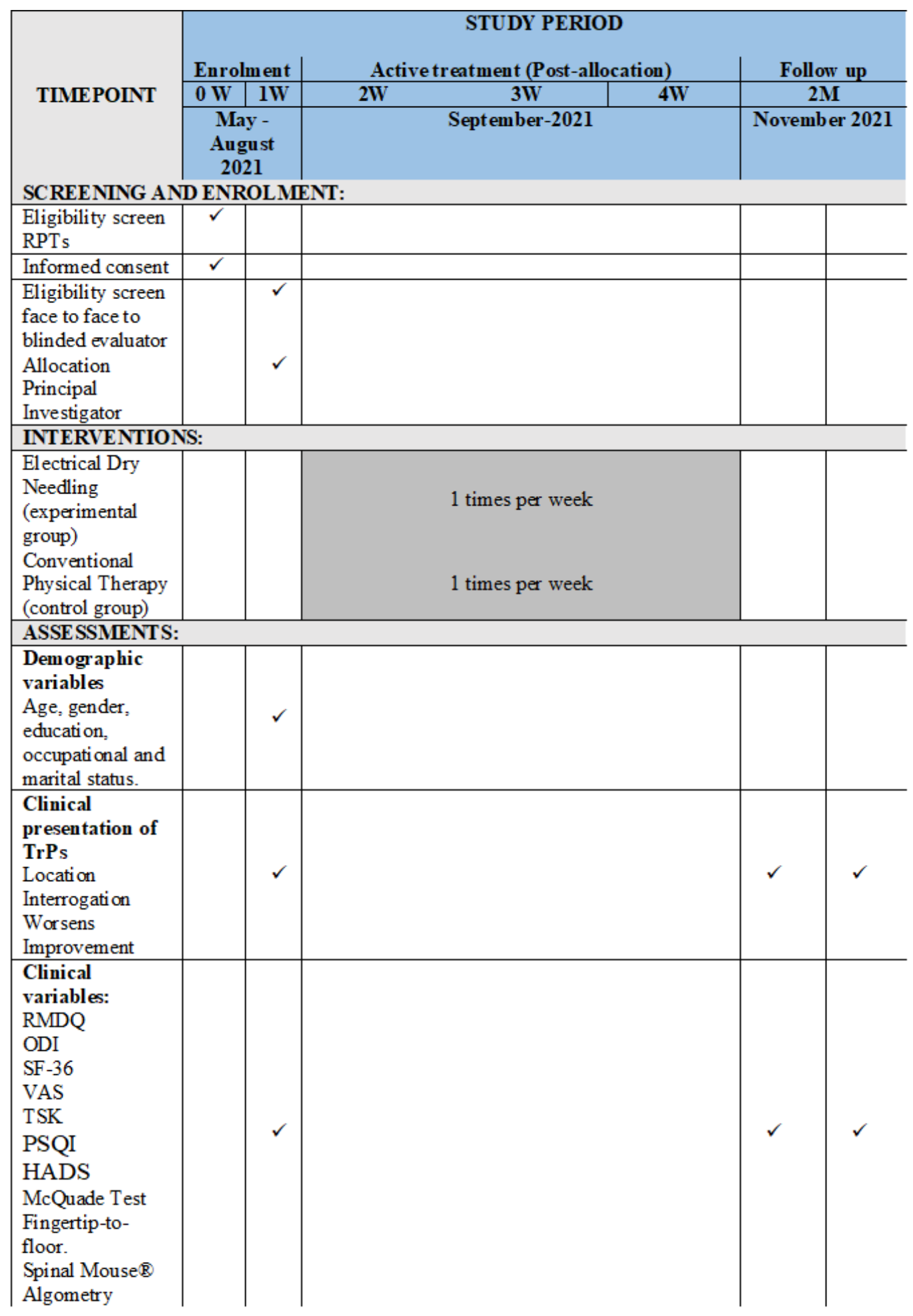

\section{Figure 3}

Timepoint of each assessment index.

\section{Supplementary Files}

This is a list of supplementary files associated with this preprint. Click to download. 
- Appendix1.Stretchingexercise..docx

- Appendix2.Homeexercise.docx

- Appendix3.INFORMEDCONSENT.docx 